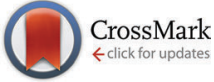

Cite this: Phys. Chem. Chem. Phys., 2015, 17, 12639

Received 8th December 2014 , Accepted 20th March 2015

DOI: $10.1039 / c 4 c p 05732 e$

www.rsc.org/pccp

\section{A molecular dynamics study of guest-host hydrogen bonding in alcohol clathrate hydrates $\dagger$}

\author{
Masaki Hiratsuka, $\stackrel{\ddagger}{\text { a }}^{\text {Ryo Ohmura, }}{ }^{a}$ Amadeu K. Sum, ${ }^{\mathrm{b}}$ Saman Alavi ${ }^{\mathrm{c}}$ and \\ Kenji Yasuoka*a
}

\begin{abstract}
Clathrate hydrates are typically stabilized by suitably sized hydrophobic guest molecules. However, it has been experimentally reported that isomers of amyl-alcohol $\mathrm{C}_{5} \mathrm{H}_{11} \mathrm{OH}$ can be enclosed into the $5^{12} 6^{4}$ cages in structure II (sIl) clathrate hydrates, even though the effective radii of the molecules are larger than the van der Waals radii of the cages. To reveal the mechanism of the anomalous enclathration of hydrophilic molecules, we performed $a b$ initio and classical molecular dynamics simulations (MD) and analyzed the structure and dynamics of a guest-host hydrogen bond for sll 3-methyl-1-butanol and structure $\mathrm{H}(\mathrm{sH})$ 2-methyl-2-butanol clathrate hydrates. The simulations clearly showed the formation of guest-host hydrogen bonds and the incorporation of the $\mathrm{O}-\mathrm{H}$ group of 3-methyl-1-butanol guest molecules into the framework of the sll $5^{12} 6^{4}$ cages, with the remaining hydrophobic part of the amyl-alcohol molecule well accommodated into the cages. The calculated vibrational spectra of alcohol $\mathrm{O}-\mathrm{H}$ bonds showed large frequency shifts due to the strong guest-host hydrogen bonding. The 2-methyl-2-butanol guests form strong hydrogen bonds with the cage water molecules in the $\mathrm{sH}$ clathrate, but are not incorporated into the water framework. By comparing the structures of the alcohols in the hydrate phases, the effect of the location of $\mathrm{O}-\mathrm{H}$ groups in the butyl chain of the guest molecules on the crystalline structure of the clathrate hydrates is indicated.
\end{abstract}

\section{Introduction}

Clathrate hydrates are crystalline inclusion compounds consisting of hydrogen bonded water molecules forming cages that are stabilized by guest-host non-covalent interactions. Huge amounts of natural gas hydrates are located in the ocean floor and considered as potential future energy resources. ${ }^{1}$ Because of high occupancy and reaction selectivity of clathrate hydrates, various other industrial applications are suggested, such as gas storage, transportation, and gas sequestration. ${ }^{2-8}$ However, hydrates are usually stable under high pressure and at low temperature and these features often inhibit practical use of clathrate hydrates. For the effective use of clathrate hydrate, a detailed understanding and controlling of the stability are significant.

Clathrate hydrates are typically stabilized by suitably sized hydrophobic guest molecules. The van der Waals-Platteeuw theory, ${ }^{9}$

\footnotetext{
${ }^{a}$ Department of Mechanical Engineering, Keio University, 3-4-1 Hiyoshi, Kohoku-ku, Yokohama 223-8522, Japan. E-mail: yasuoka@mech.keio.ac.jp

${ }^{b}$ Center for Hydrate Research, Department of Chemical \& Biological Engineering, Colorado School of Mines, Golden, CO 80401, USA

${ }^{c}$ Center for Catalysis Research and Innovation, Department of Chemistry,

University of Ottawa, Ottawa, Ontario KIN 7N5, Canada

$\dagger$ Electronic supplementary information (ESI) available. See DOI: 10.1039/c4cp05732e \$ Current address: Department of Mechanical Engineering, Kogakuin University, 2665-1 Nakano, Hachioji, Tokyo 192-0015, Japan.
}

which mainly considers guest-host van der Waals interactions, is commonly used to estimate the phase equilibrium conditions of clathrate hydrates. ${ }^{10}$ Hydrophilic molecules like methanol, ethanol, and ammonia form hydrogen bonds with water and are historically considered thermodynamic hydrate inhibitors. ${ }^{11,12}$ However, recent experimental observations ${ }^{13-17}$ reported the formation, not inhibition, of clathrate hydrates with these hydrophilic guest molecules, which actually even show promoting effects. ${ }^{18-20}$ Methanol and ammonia clathrate hydrates are also considered as components of the astronomical bodies, such as in comets and Saturn's moons, Titan and Enceladus. ${ }^{13,19,21}$ In clathrate hydrates enclosing hydrophilic molecules, the formation of a guest-host hydrogen bond is experimentally observed by powder and single X-ray diffraction, ${ }^{22-25}$ FTIR and Raman measurements. ${ }^{16,26}$ Molecular dynamics (MD) simulations of these systems show that guest-host hydrogen bonds induce Bjerrum defects in the water lattice hydrogen bond network and affect the molecular motion of the guest molecules. ${ }^{15,27-31}$ In these experimental and computational studies, the disorder of the water lattice induced by the guest-host hydrogen bond and dynamics of the guest molecules in the cages is revealed. This type of strong molecular interaction also exists in clathrate hydrates containing ionic species, ${ }^{17,32}$ halogen molecules, ${ }^{33,34}$ and polar molecules ${ }^{35}$ as guests and is reported to induce changes in the structures of the water lattice and occupancy of the guest substance. To make 
use of the effects induced by hydrophilic molecules and their impact on the thermodynamics of the clathrate, a more detailed understanding of the microscopic structures and molecular interactions of the guest-host molecules is important.

In this study, we focus on amyl-alcohol isomers $\mathrm{C}_{5} \mathrm{H}_{11} \mathrm{OH}$ as guests in clathrate hydrate phases. One of the amyl-alcohol isomers, 3-methyl-1-butanol, is enclosed in the $5^{12} 6^{8}$ cages of sH hydrate with xenon as help gas in the $5^{12}$ and $4^{3} 5^{6} 6^{3}$ cages. ${ }^{36}$ A recent X-ray observation ${ }^{18}$ reported that this molecule can also be enclosed in the $5^{12} 6^{4}$ cages in sII hydrates with methane as help gas in the $5^{12}$ cages. However, the length of the longest dimension of 3-methyl-1-butanol in a vacuum determined by DFT computations [B3LYP/6-311++G(d,p)] is $9.04 \AA^{16}$ This length is considered to be too long for the molecule to fit in the $5^{12} 6^{4}$ cage of sII, where the length of the major axis is $6.29 \AA$ An In comparison, the major axis of the $5^{12} 6^{8}$ cage in $\mathrm{sH}$ hydrates is $8.44 \AA^{37}$ This enclathration of alcohol molecules is difficult to understand within the framework of the previous theory of the cage stability containing hydrophobic molecules. To understand this anomalous enclathration of a large molecule in the sII hydrate, Cha et al. synthesized clathrate hydrates containing eight amyl-alcohol isomers with methane as help gas and determined their structures. ${ }^{16}$ They found that amylalcohol isomers 3-methyl-1-butanol (9.04 $\AA$ end-to-end length in vacuum) and 2-2-dimethyl-1-propanol (an end-to-end length of $7.76 \AA$ in a vacuum) form sII hydrates, while amyl-alcohol isomers, 2-methyl-2-butanol (an end-to-end length of $7.76 \AA$ in a vacuum) and 3-methyl-2-butanol (an end-to-end length of $8.01 \AA$ in a vacuum) form sH clathrate hydrates. The results were determined from Raman spectra measurements of the hydrate phases and observation that the free 3-methyl-1-butanol $\mathrm{O}-\mathrm{H}$ stretching mode found in $\mathrm{sH}$ hydrates was not detected in the sII hydrate. It was concluded that the $\mathrm{O}-\mathrm{H}$ groups of the amylalcohols are incorporated into the host cages to fit the relatively small $5^{12} 6^{4}$ cage in the sII hydrate. These results showed the significant effect of guest-host hydrogen bonding on the structure of the clathrate hydrate formed. To provide more detailed and direct insight of the guest-host hydrogen bond in clathrate hydrate, we performed MD simulation of amyl-alcohol molecules in sII (3-methyl-1-butanol + methane) and sH (2-methyl-2butanol + methane) clathrate hydrates. For the characterization of the hydrogen bond and accurate computation of the shift of the intramolecular vibrational frequencies, quantum mechanical calculations of the hydrate phase were performed. Since the observation of the dynamics of guest-host hydrogen bonding is difficult on the time scale of $a b$ initio MD simulations, we also performed classical MD simulations and analyzed the formation and lifetime of guest-host hydrogen bonds in clathrate hydrates.

\section{Computational methods}

\subsection{Ab initio MD simulation}

To analyze the guest-host hydrogen bonding and vibrational spectra of the $\mathrm{O}-\mathrm{H}$ group in the clathrate hydrate, ab initio MD simulations were performed on a single unit cell of the binary (3-methyl-1-butanol + methane) sII hydrate and two unit cells of the binary (2-methyl-2-butanol + methane) $\mathrm{sH}$ hydrate. To perform sufficiently long simulations to ensure resolution of the vibrational spectra, the Car-Parrinello $\mathrm{MD}^{38}$ simulation using density functional theory (DFT) in the CPMD package ${ }^{39}$ was employed as the $a b$ initio $\mathrm{MD}$ method. The $\mathrm{PBE}^{40}$ approximation, which is employed in the previous computation to calculate vibrational spectra of methane clathrate hydrates, ${ }^{41}$ was employed for the exchange-correlation terms in DFT. The dispersion interaction between guest and host molecules is important to calculate the properties of clathrate hydrates ${ }^{42,43}$ In this study the empirical Grimme correction DFT-D $2^{44}$ for the dispersion force was applied. The valence core interaction was described by Troullier-Martins ${ }^{45}$ and norm-conserving pseudopotential using the Kleinman-Bylander ${ }^{46}$ separation scheme for carbon, hydrogen, and oxygen. The energy cutoff of the plane waves was 80 Ry and the Brillouin zone of the supercell was sampled at the $\Gamma$ point. The simulation time-step was $0.097 \mathrm{fs}$ and the fictitious electron mass was 400 a.u. for the Car-Parrinello method. Although in Car-Parrinello MD a redshift in the dynamics of light atoms due to the fictitious mass of the electron dynamics is recognized, ${ }^{47}$ the redshift has little effect on the comparison of the differences of the vibrational frequencies of the guest alcohol molecules between cages. ${ }^{48,49}$

The sII clathrate hydrate is cubic and composed of sixteen $5^{12}$ cages and eight $5^{12} 6^{4}$ cages formed by 136 water molecules. In this calculation, 3-methyl-1-butanol and methane molecules occupied all $5^{12} 6^{4}$ and $5^{12}$ cages, respectively. The unit cell of $\mathrm{sH}$ clathrate hydrates is hexagonal and composed of three $5^{12}$, two $4^{3} 5^{6} 6^{3}$, and one $5^{12} 6^{8}$ cages with 34 water molecules per unit cell. The 2-methyl-2-butanol guest occupies the $5^{12} 6^{8}$ cages and methane the $5^{12}$ and $4^{3} 5^{6} 6^{3}$ cages of the sH hydrate. Since the rotational relaxation time of the guest molecule is much larger than the time scale of the ab initio MD simulation, the initial configurations are generated by the classical MD simulations explained in the next section. The vibrational spectra of the guest molecules were computed by the Fourier transform of the velocity autocorrelation function of the hydrogen atom in the $\mathrm{O}-\mathrm{H}$ group of the alcohol molecules computed from the MD simulation trajectories. The total simulation time was $9 \mathrm{ps}$. The initial 1 ps was used for the equilibration and the remaining 8 ps were used to average the physical values. The velocity autocorrelation function was calculated over $4 \mathrm{ps}$ with a total of 200 autocorrelation functions calculated every $20 \mathrm{fs}$ and then averaged. The resolution of the vibrational spectra was $8.64 \mathrm{~cm}^{-1}$. The Hann window function was employed for the Fourier transforms. In the initial 1 ps of equilibration, the velocity scaling method was employed to control the temperature to $113 \mathrm{~K}$. The other 8 ps used to calculate the autocorrelation functions were performed in the constant NVE ensemble.

\subsection{Classical MD simulation}

Classical MD simulations were performed on $2 \times 2 \times 2$ replicas of the sII hydrate unit cells (1088 water molecules) and $3 \times 4 \times 4$ replicas of $\mathrm{sH}$ hydrate unit cells (1632 water molecules) to calculate the probability and dynamical properties of hydrogen 
bonds in the amyl-alcohol clathrate hydrates. Initial positions of oxygen atoms in the clathrate hydrate unit cell are those obtained by X-ray diffraction ${ }^{50}$ and proton configurations are determined to satisfy the ice rule, zero net-dipole moment and minimum potential energy. ${ }^{51}$ The GROMACS ${ }^{52,53}$ computational package was used to generate trajectories of clathrate hydrates at the respective temperatures. The TIP4P/ice ${ }^{54}$ water model, which is known to give reasonable prediction of phase diagrams of clathrate hydrates, ${ }^{55}$ was used in the simulations. The flexible general AMBER force field ${ }^{56}$ (GAFF) was used for 3-methyl-1-butanol, 2-methyl-2-butanol, and methane molecules. The partial charge distribution of the amyl-alcohols and methane molecules was determined by the ChelpG method at the MP2/ aug-cc-pVTZ level of theory with Gaussian $03^{57}$ on the optimized structure with MP2/6-311++G(d,p). Simulations were performed for $10 \mathrm{~ns}$ with a small time step of $0.2 \mathrm{fs}$, using the NPT ensemble with Nosé-Hoover and Parrinello-Rahman algorithms for the thermostat and barostat, respectively. The simulation conditions were set to be different temperatures $(113 \mathrm{~K}, 200 \mathrm{~K}, 270 \mathrm{~K})$ and a pressure of $6 \mathrm{MPa}$. The initial $1 \mathrm{~ns}$ of the simulation was used for equilibration and remaining $9 \mathrm{~ns}$ were used for the calculation of the statistical average of the properties. The short range cutoff was $14 \AA$ and the calculation of the Coulomb potential was performed with the particle mesh Ewald (PME) method.

\section{Results and discussion}

Fig. 1 shows snapshots of the 3-methyl-1-butanol and 2-methyl2-butanol molecules enclosed in the large cages of sII and $\mathrm{sH}$ hydrates, respectively, obtained from the $a b$ initio MD simulations. In the $5^{12} 6^{4}$ cages of sII, strong guest-host hydrogen bonds are formed between 3-methyl-1-butanol and water molecules, observed in both $a b$ initio and classical MD simulations. Furthermore, in many of the $5^{12} 6^{4}$ cages, the $\mathrm{O}-\mathrm{H}$ groups of the alcohol molecules were inserted between two water molecules in the hydrogen bonding network of the cages. The $\mathrm{O}-\mathrm{H}$ group of 3-methyl-1-butanol in the $5^{12} 6^{4}$ cages pushes open the wall of the cages as shown in Fig. 1(a) and (b). This structural feature has been previously observed in other alcohol guest molecules in hydrate phases. ${ }^{29,58}$

The probability of the hydrogen bonding computed from $10 \mathrm{~ns}$ of the classical MD simulation is shown in Table 1. The probability of the formation of hydrogen bonds with $i$ molecules $P_{i}(i=1,2$ or none 0$)$ is defined as

$$
P_{i}=\frac{N_{i}}{N_{\text {tot }}}
$$

where $N_{i}$ is the number of guest molecules forming hydrogen bonds with $i$ molecules. $N_{\text {tot }}$ is simply the total number of guest molecules. The formation of the hydrogen bond was assigned by the $\mathrm{H} \cdots \mathrm{B}$ distance in the $\mathrm{A}-\mathrm{H} \cdots \mathrm{B}$ bonding, less than $2.4 \AA$, and the $\mathrm{A}-\mathrm{H} \cdots \mathrm{B}$ angle, greater than $150^{\circ}$.

The $\mathrm{RO}-\mathrm{H}_{\mathrm{g}} \cdots \mathrm{O}_{\mathrm{w}}$ guest-host hydrogen bond in the sII hydrate of 3-methyl-1-butanol was formed in $98 \%$ and $85 \%$ of the trajectory time at $113 \mathrm{~K}$ and $270 \mathrm{~K}$, respectively. These probabilities are higher than other alcohol molecules such as ethanol, 1-propanol
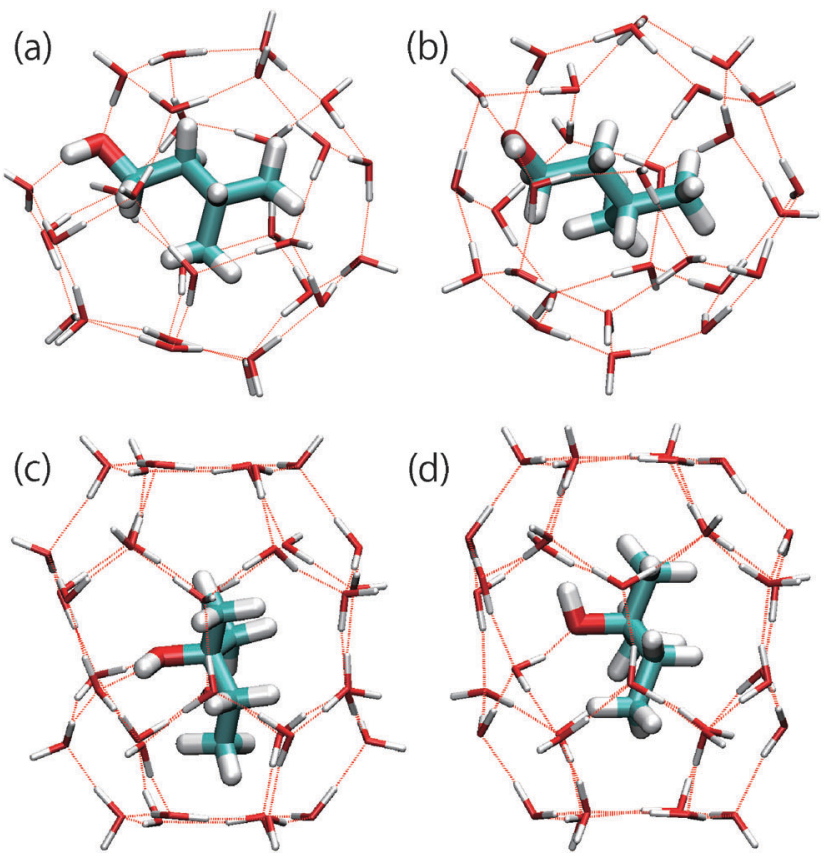

(d)

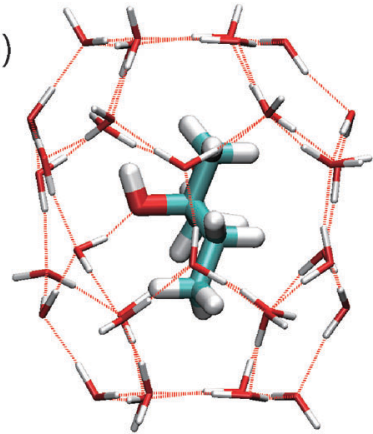

Fig. 1 Snapshots of the $5^{12} 6^{4}$ cages in sll and $5^{12} 6^{8}$ cages in $\mathrm{sH}$ clathrate hydrates from the ab initio MD simulations. The guest-host hydrogen bonds are observed in both structures. The $\mathrm{O}-\mathrm{H}$ group of 3-methyl-1butanol molecules in sll hydrates is incorporated into $5^{12} 6^{4}$ cages and forms hydrogen bonds with (a) two or (b) three water molecules. The $\mathrm{O}-\mathrm{H}$ group of 2-methyl-2-butanol molecules in $\mathrm{sH}$ hydrates forms hydrogen bonds with (c) two or (d) one water molecules. The $\mathrm{H}$ atom in the alcohol $\mathrm{O}-\mathrm{H}$ group is free from the hydrogen bonding in the snapshot (d).

Table 1 Averaged probability of hydrogen bonds

\begin{tabular}{|c|c|c|c|c|c|}
\hline \multirow[b]{2}{*}{ Temp./K } & \multirow[b]{2}{*}{$\mathrm{N}$ bond } & \multicolumn{2}{|c|}{$\begin{array}{l}\text { 3-Methyl-1-butanol } \\
\text { in sII } 5^{12} 6^{4}\end{array}$} & \multicolumn{2}{|c|}{$\begin{array}{l}\text { 2-Methyl-2-butanol } \\
\text { in sH } 5^{12} 6^{8}\end{array}$} \\
\hline & & $\mathrm{H}_{\mathrm{g}} \cdots \mathrm{O}_{\mathrm{w}}$ & $\mathrm{O}_{\mathrm{g}} \cdots \mathrm{H}_{\mathrm{w}}$ & $\mathrm{H}_{\mathrm{g}} \cdots \mathrm{O}_{\mathrm{w}}$ & $\mathrm{O}_{\mathrm{g}} \cdots \mathrm{H}_{\mathrm{w}}$ \\
\hline \multirow[t]{3}{*}{113} & 0 & 0.017 & 0.004 & 0.147 & 0.022 \\
\hline & 1 & 0.983 & 0.597 & 0.853 & 0.958 \\
\hline & 2 & - & 0.399 & - & 0.020 \\
\hline \multirow[t]{3}{*}{200} & 0 & 0.054 & 0.018 & 0.291 & 0.052 \\
\hline & 1 & 0.946 & 0.680 & 0.709 & 0.932 \\
\hline & 2 & - & 0.302 & - & 0.016 \\
\hline \multirow[t]{3}{*}{270} & 0 & 0.152 & 0.057 & 0.430 & 0.114 \\
\hline & 1 & 0.848 & 0.691 & 0.570 & 0.882 \\
\hline & 2 & - & 0.252 & - & 0.005 \\
\hline
\end{tabular}

and 2-propanol in the sII clathrate hydrates calculated in previous classical MD simulations, which formed with about $83 \%$ and $48 \%$ probability at $100 \mathrm{~K}$ and $250 \mathrm{~K}$, respectively. ${ }^{29}$ In the $a b$ initio MD simulation at $113 \mathrm{~K}$, all eight 3-methyl-1-butanol molecules in the unit cell formed hydrogen bonds with water molecules during the 8 ps simulation time. This high probability of guest-host hydrogen bonding shows the contribution of guest-water hydrogen bonding in stabilizing the hydrate structure, which is also evidenced in previous studies. ${ }^{16}$ The guest-host hydrogen bond allows the $\mathrm{O}-\mathrm{H}$ group of 3-methyl-1-butanol to be incorporated into the cages and the hydrophobic part to fit into the $5^{12} 6^{4}$ cage. Without this particular form of hydrogen bonding, the amyl-alcohol guest 


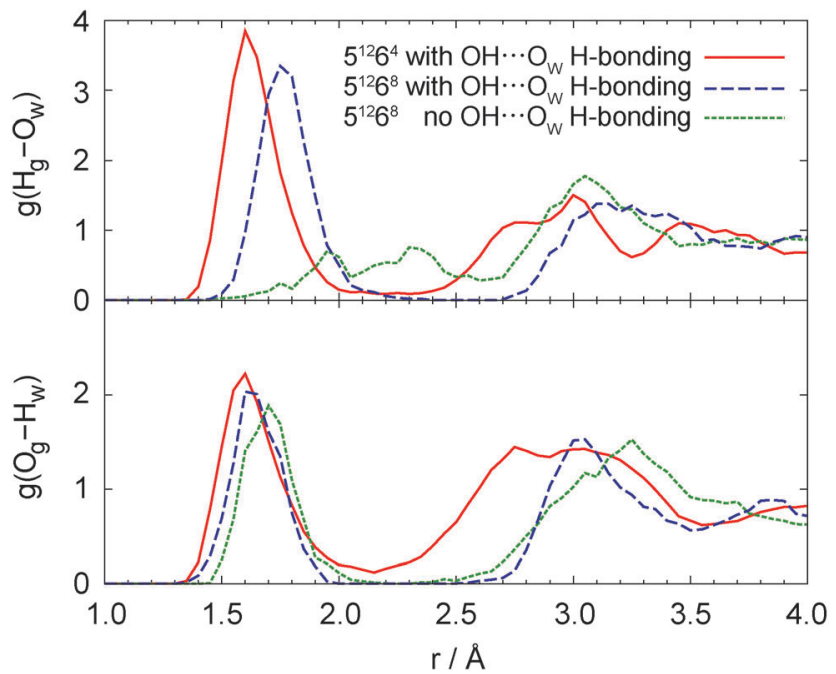

Fig. 2 Radial distribution function for the $\mathrm{H}$ atom in the $\mathrm{O}-\mathrm{H}$ group of the guest alcohol molecule to the $\mathrm{O}$ atom in the water molecules $\left(\mathrm{H}_{\mathrm{g}} \cdots \mathrm{O}_{\mathrm{w}}\right.$; top) and the $\mathrm{O}$ atom in the $\mathrm{O}-\mathrm{H}$ group of guest alcohol molecules to $\mathrm{H}$ atom in the water molecules $\left(\mathrm{O}_{\mathrm{g}} \cdots \mathrm{H}_{\mathrm{w}}\right.$; bottom) in cages calculated by ab initio MD simulations. The length of the hydrogen bond in the $5^{12} 6^{4}$ cages in the sll hydrates is smaller than that in the $5^{12} 6^{8}$ cages in $\mathrm{sH}$ hydrates. The smaller distance reflects a strong hydrogen bond and caused the large frequency shift shown in Fig. 4(a).

molecule would be too large to fit into the $5^{12} 6^{4}$ sII hydrate cage. The oxygen atoms of the alcohol molecules act as one or two hydrogen bond proton acceptors from the cage water molecules. The alcohol O-H groups inserted into both five- and six-member water rings in the cages. Configurations where the guest alcohol $\mathrm{O}$ atom $\left(\mathrm{O}_{\mathrm{g}}\right)$ hydrogen bonds with the $\mathrm{H}$ atoms $\left(\mathrm{H}_{\mathrm{w}}\right)$ of two water molecules were most important in the lower temperature simulations, as shown in Table 1.

Fig. 2 and 3 show the radial distribution function (RDF) for the hydrogen atom in the alcohol $\mathrm{O}-\mathrm{H}$ group to the oxygen atoms in the cage water molecules calculated from the trajectory of $a b$ initio $\mathrm{MD}$ and classical MD simulations.

The first peak of the RDF is at $1.63 \AA$ and $1.69 \AA$ from the $a b$ initio and classical MD simulations, respectively. These distances are smaller than the previous observation by classical MD, about $1.8 \AA$ for ethanol and 2-propanol molecules, $1.78 \AA$ for 1-propanol, ${ }^{29}$ and $2.2 \AA$ in tert-butylamine ${ }^{59}$ in the $5^{12} 6^{4}$ cage in sII hydrate. On the other hand, the first peak of the RDF for the oxygen atom in the alcohol $\mathrm{O}-\mathrm{H}$ group to the hydrogen atom in water molecules $\left(\mathrm{O}_{\mathrm{g}} \cdot \mathrm{H}_{\mathrm{w}}\right)$ is at $1.7 \AA$ at all temperatures and structures from classical MD simulations. This distance for the $\left(\mathrm{O}_{\mathrm{g}} \cdot \mathrm{H}_{\mathrm{w}}\right)$ hydrogen bond is similar to the corresponding hydrogen bonding length in ethanol, 1-propanol, 2-propanol ${ }^{29}$ and tert-butylamine molecules in the sII hydrate ${ }^{59}(\sim 1.7 \AA)$. The difference of the hydrogen bond length mainly appears in the $\mathrm{H}_{\mathrm{g}} \cdots \mathrm{O}_{\mathrm{w}} \mathrm{RDF}$.

Fig. 4(a) shows the calculated vibrational spectra of the hydrogen atoms in the $\mathrm{O}-\mathrm{H}$ groups of 3-methyl-1-butanol molecules in the $5^{12} 6^{4}$ cage of sII hydrate calculated by ab initio MD. The calculated vibrational spectrum of the isolated alcohol molecule in a vacuum is also shown for comparison. The vibrational

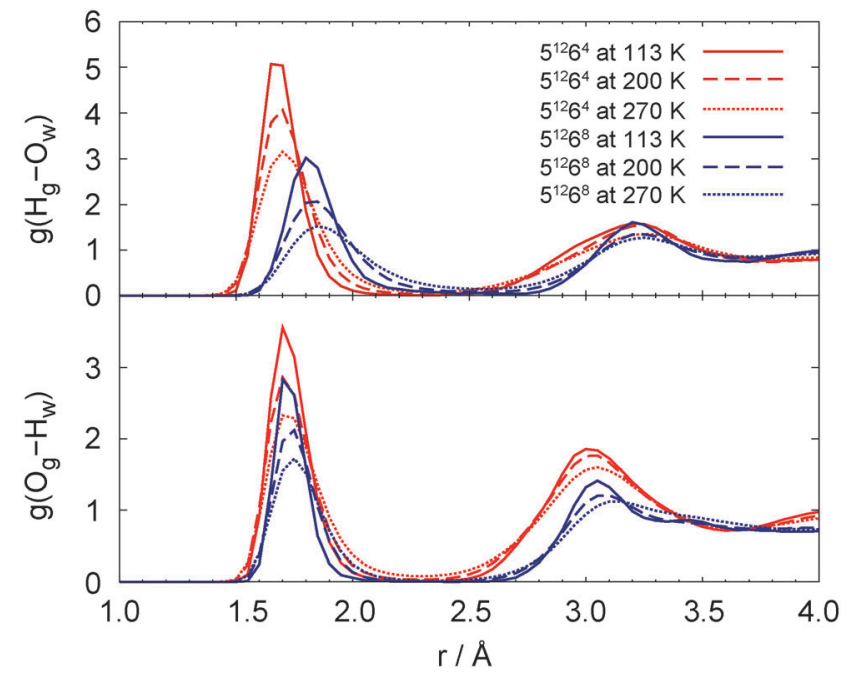

Fig. 3 Radial distribution function for the $\mathrm{H}$ atom in the $\mathrm{O}-\mathrm{H}$ group of the guest alcohol molecules to the $\mathrm{O}$ atom in the water molecules $\left(\mathrm{H}_{\mathrm{g}} \cdots \mathrm{O}_{\mathrm{w}}\right.$; top) and the $\mathrm{O}$ atom in the $\mathrm{O}-\mathrm{H}$ group of the guest alcohol molecules to the $\mathrm{H}$ atom in the water molecules $\left(\mathrm{O}_{\mathrm{g}} \cdot \mathrm{H}_{\mathrm{w}}\right.$; bottom) calculated by classical MD simulations. The length of the $\mathrm{H}_{\mathrm{g}} \cdots \mathrm{O}_{\mathrm{w}}$ hydrogen bond in the $5^{12} 6^{4}$ cages in the sll hydrates is smaller than that in the $5^{12} 6^{8}$ cages in $\mathrm{sH}$ hydrates. On the other hand, the length of the $\mathrm{O}_{\mathrm{g}} \cdots \mathrm{H}_{\mathrm{w}}$ hydrogen bond is identical in all systems. The tail of the first peak in the $5^{12} 6^{8}$ cages (blue lines) shows the non-hydrogen bonded amyl-alcohol molecules in the $\mathrm{sH}$ hydrate, corresponding to the free $\mathrm{O}-\mathrm{H}$ vibrational spectra.

peak of the stretching vibrational mode of the free $\mathrm{O}-\mathrm{H}$ for an isolated molecule is located at $3500 \mathrm{~cm}^{-1}$. In comparison, the vibrational peak of the hydrogen bonded $\mathrm{O}-\mathrm{H}$ in the cages is shifted to lower frequency, in the range between $2800-3400 \mathrm{~cm}^{-1}$, along with a broadening in the peak shape. This result supports the interpretation of the previous experimental Raman observation of amyl-alcohols in the sII hydrate, ${ }^{16}$ in that the undetected peak of the free $\mathrm{O}-\mathrm{H}$ stretching vibration in the sII hydrate is caused by the guest-host hydrogen bond and red shift of the $\mathrm{O}-\mathrm{H}$ stretching frequency. The amyl-alcohol molecules in sII hydrates form hydrogen bonds with water molecules forming the cages with high probability and as a consequence, the vibrational modes of the amyl-alcohol molecules are drastically altered.

In the sH 2-methyl-2-butanol hydrate, the probability of the hydrogen bond was smaller than that in the sII hydrate enclosing 3-methyl-1-butanol $\left(\mathrm{H}_{\mathrm{g}} \cdots \mathrm{O}_{\mathrm{w}}\right.$ in Table 1). In this case, about $85 \%$ of hydrogen atoms of $\mathrm{O}-\mathrm{H}$ groups $\left(\mathrm{H}_{\mathrm{g}}\right)$ in the 2-methyl-2-butanol molecules form guest-host hydrogen bonds in the cages of $\mathrm{sH}$, but $15 \%$ of the $\mathrm{H}_{\mathrm{g}}$ atoms did not form any guest-host hydrogen bonds even at $113 \mathrm{~K}$ as shown in Fig. 1(c) and (d), respectively. The oxygen atoms in the alcohol $\mathrm{O}-\mathrm{H}$ form the guest-host hydrogen bondings in higher probability than the hydrogen atoms, about 95\%. Fig. 4(b) shows the vibrational spectra of the hydrogen atoms in $\mathrm{O}-\mathrm{H}$ groups of 2-methyl-2-butanol molecules in the $5^{12} 6^{8}$ cage of $\mathrm{sH}$ hydrate calculated from the $a b$ initio MD simulations. The red solid line is the vibrational spectrum of selected alcohol molecules in $\mathrm{sH}$ hydrate forming a guest-host hydrogen bond as shown in the snapshot in Fig. 1(c). The hydrogen bonded $\mathrm{O}-\mathrm{H}$ stretching 


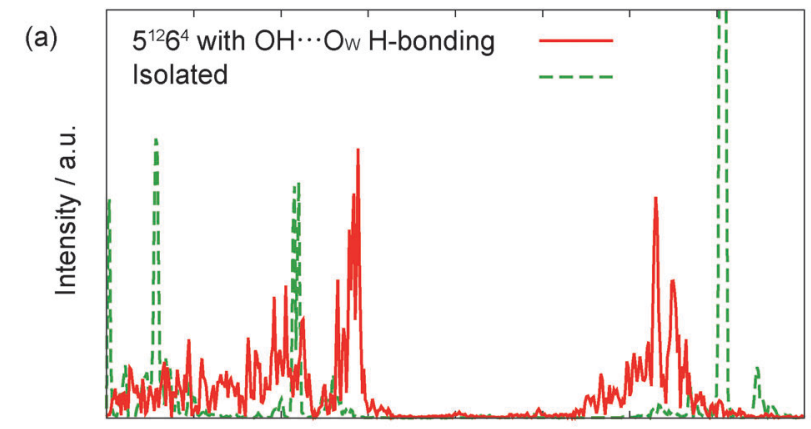

(b)

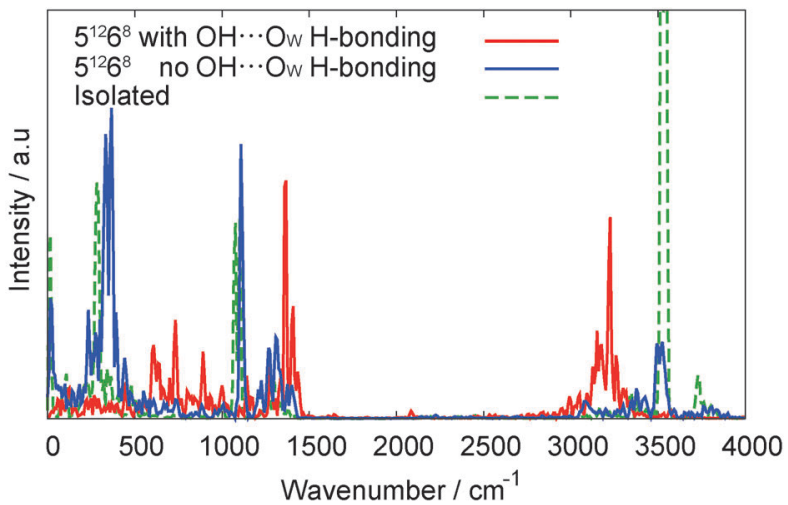

Fig. 4 (a) Vibrational spectra of 3-methyl-1-butanol molecules in a vacuum (green dashed lines) and in the sll clathrate hydrates computed by the Fourier transform of the velocity autocorrelation function of the $\mathrm{H}$ atom in the $\mathrm{O}-\mathrm{H}$ group from ab initio MD simulations (red line). The $\mathrm{O}-\mathrm{H}$ stretching vibrational peak located at $3500 \mathrm{~cm}^{-1}$ in a vacuum shifted to lower frequency and also broadened due to the hydrogen bonding between guest and water molecules. (b) Vibrational spectra of 2-methyl2 -butanol molecules in a vacuum and in the $\mathrm{sH}$ hydrate. The red and blue solid lines are from the guest molecule that formed and not formed $\mathrm{H}_{\mathrm{g}} \cdots \mathrm{O}_{\mathrm{w}}$ hydrogen bonding, respectively. (shown in Fig. 1(c) and (d)).

vibrational peak of 2-methyl-2-butanol in the $\mathrm{sH}$ hydrates is shifted to lower frequency $\left(3000-3300 \mathrm{~cm}^{-1}\right)$. The shift is smaller than that in the sII hydrate containing 3-methyl-1butanol. The blue line is the vibrational spectrum of a selected 2-methyl-2-butanol molecule in a $\mathrm{sH}$ cage that has no $\mathrm{H}_{\mathrm{g}} \cdots \mathrm{O}_{\mathrm{w}}$ guest-host hydrogen bond as shown in Fig. 1(d). The frequency of the $\mathrm{O}-\mathrm{H}$ bond in 2-methyl-2-butanol molecule with no $\mathrm{H}_{\mathrm{g}} \cdots \mathrm{O}_{\mathrm{w}}$ hydrogen bond in the cage is similar to an uncaged molecule. The RDF for ROH to $\mathrm{O}_{\mathrm{w}}$ is shown in Fig. 2 and 3. The length of the guest-host hydrogen bond in sH is larger $(0.15 \AA)$ than in SII and the peak shift of the $\mathrm{O}-\mathrm{H}$ stretching vibration was less than that in the sII hydrate. This result suggests that the guest-host hydrogen bond can be formed in the $5^{12} 6^{8}$ cage but is weaker than that in the sII hydrate enclosing 3-methyl-1butanol. This seemingly weaker hydrogen bonding in the $\mathrm{sH}$ hydrate is consistent with the observations of the hydrogen bonding strength of pinacolone and tert-butylmethylether in the $\mathrm{sH}$ clathrate hydrate. ${ }^{59}$

Here we consider structural reasons why different sII and $\mathrm{sH}$ structures are obtained for the different amyl-alcohol isomers. Even though the longest large atom distance in 2-methyl-2butanol, 7.76 $\AA$, is smaller than that in 3-methyl-1-butanol, 9.01 $\AA$, only the enclathration of the latter molecule in the $5^{12} 6^{4}$ cage in
sII was observed. ${ }^{16}$ The difference in hydrate structures primarily arises from the difference in the location of the $\mathrm{O}-\mathrm{H}$ group in the amyl-alcohol guest molecules. To enclose the 3-methyl-1-butanol molecule into the relatively small $5^{12} 6^{4}$ cage in sII, the incorporation of the $\mathrm{O}-\mathrm{H}$ group by the guest-host hydrogen bond is required. The $\mathrm{O}-\mathrm{H}$ group of the 3-methyl-1-butanol is located at the end of the molecules and is not encumbered by nearby methyl groups. Therefore the $\mathrm{O}-\mathrm{H}$ group can easily hydrogen bond with water molecules in the cages and even be inserted among the water molecules. As a result, 3-methyl-1-butanol can be incorporated into the relatively smaller $5^{12} 6^{4}$ cages in the sII hydrate. The $\mathrm{O}-\mathrm{H}$ group of 2-methyl-2-butanol is adjacent to two methyl groups, which cause steric repulsions with cage water molecules when forming hydrogen bonds with the cage waters. As such, the O-H group of 2-methyl-2-butanol cannot as easily be incorporated into the hydrogen bonding network of the cage water molecules. A similar trend is also observed in other amylalcohol molecules discussed in the previous experimental work, ${ }^{16}$ namely, 2-2-dimethyl-1-propanol forming sII and 3-methyl-2butanol forming sH hydrate. As shown here, the structure of the clathrate hydrates forming guest-host hydrogen bonds can strongly be affected by the microscopic structure of the guest molecule, including the position of the alcohol $\mathrm{O}-\mathrm{H}$ groups and the size of the molecules.

The other significant factor on the stability of the clathrate hydrates is the conformers of the guest molecules. Recently Frankcombe and Kroes calculated the formation energies of the clathrate hydrates and showed the significant impact of the gauche configurations of the guest molecules on the hydrate stability. ${ }^{60}$ In the case of the butane molecules in the sII $5^{12} 6^{4}$ cages, the gauche conformation is more favorable than the trans conformation. To understand the relation between the conformers and the structure of the clathrate hydrates, we calculated the probability of the conformers of the alcohol guest molecules. The calculated probability of the gauche structure at $270 \mathrm{~K}$ was $40.9 \%$ in 3-methyl-1-butanol molecules in the sII $5^{12} 6^{4}$ and $4.41 \%$ in the 2-methyl-2-butanol molecules in the $\mathrm{sH} 5^{12} 6^{8}$ cages. The length of the gauche conformation of the 3-methyl-1-butanol molecule is smaller than the trans conformation of the molecule. Therefore the half of the 3-methyl-1-butanol molecules is compactly packed as the gauche structure in the sII hydrate to fit the size of the $5^{12} 6^{4}$ cages. On the other hand, most of the 2-methyl-2butanol molecules in the $\mathrm{sH} 5^{12} 6^{8}$ cages form a trans structure. As such, the 3-methyl-1-butanol molecules are fitted to the $5^{12} 6^{2}$ cages by the guest-host hydrogen bonding and forming the gauche conformation.

Another possibility for the incorporation of 3-methyl-1butanol molecules into the sII hydrate lattice is the displacement of a water molecule by the alcohol $\mathrm{O}-\mathrm{H}$ group. The incorporation of the alcohol $\mathrm{O}-\mathrm{H}$ groups as part of the water cages is possible and would retain the 28 oxygen vertices of the $5^{12} 6^{4}$ cage. However, this would introduce Bjerrum L-defects into the hydrogen bonding network, and make the cage structures more disordered. In one simulation, we artificially removed a water molecule from the hydrate lattice and computed the vibrational spectra of 3-methyl-1butanol with ab initio MD. Fig. 5 shows the $\mathrm{O}-\mathrm{H}$ vibrational spectra 


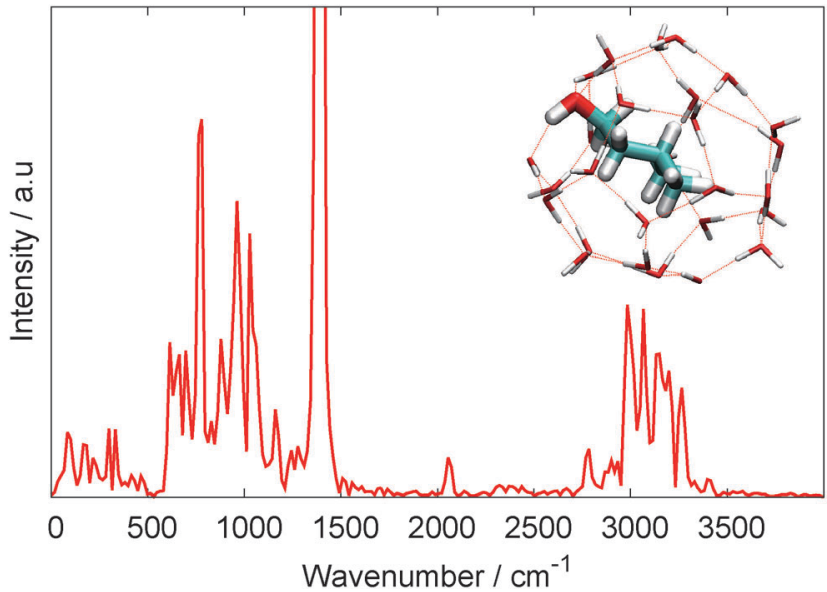

Fig. 5 Vibrational spectrum and snapshot of a 3-methyl-1-butanol molecule replacing a water molecule in the $5^{12} 6^{4}$ cage. The spectrum was computed from the $\mathrm{H}$ atom in the $\mathrm{O}-\mathrm{H}$ group of 3-methyl-1-butanol molecules. The initial structure was artificially generated by removing one water molecule from the cage. The characteristics of the vibrational spectrum was very similar to the configuration with an $\mathrm{O}-\mathrm{H}$ group inserted between the hydrogen bond of two water molecules shown in Fig. 4 a.

and the snapshot of the 3-methyl-1-butanol molecule replacing a water molecule in the $5^{12} 6^{4}$ cage in the sII hydrate. There are no drastic differences in the vibrational spectra of the 3-methyl-1butanol incorporated into the cages as compared to the case where the $\mathrm{O}-\mathrm{H}$ bond is inserted into the hydrogen bonded network, therefore it is difficult to detect this configuration using Raman spectroscopy.

The guest-host hydrogen bondings affect the rotation of the guest molecules in the cages. ${ }^{28,29,59}$ To determine the effect of the guest-host hydrogen bonds on motion of the guest molecules, the rotation of the unit vector in the direction of the alcohol $\mathrm{O}-\mathrm{H}$ group of guest molecules, $\mu$, was calculated from classical MD simulation. The rotational motion of a guest molecule at a time $t$ after some origin is characterized by the rotation of this unit vector,

$$
\cos \theta(t)=\mu(0) \cdot \mu(t)
$$

Fig. 6 shows the second Legendre polynomial $M_{2}(t)$ for the cosine of the rotation angle of the $\mathrm{O}-\mathrm{H}$ bond, for 3-methyl-1butanol in the sII hydrate and 2-methyl-2-butanol in the $\mathrm{sH}$ hydrate. The $M_{2}(t)$ function is closely linked with relaxation times in NMR spectroscopy. ${ }^{61,62}$ The $M_{2}(t)$ decays faster at higher temperatures as the alcohol guests gain greater rotational freedom in the cages. The relaxation times of intramolecular geometry changes are known to have a relaxation time of $\sim 1 \mathrm{ps}^{28,29,59}$ and molecular rotations have larger relaxation time.

$$
M_{2}(t)=\frac{1}{2}\left\langle 3 \cos ^{2} \theta(t)-1\right\rangle
$$

Despite the higher probability of guest-host hydrogen bonding in the $5^{12} 6^{4}$ cages of sII as shown in Table 1 , the relaxation of the $M_{2}(t)$ curves for 3-methyl-1-butanol in the $5^{12} 6^{4}$ cages is more quick than that of 2-methyl-2-butanol in the larger $5^{12} 6^{8}$ of the $\mathrm{sH}$ cages (the relaxation times of $M_{2}(t)$ determined by

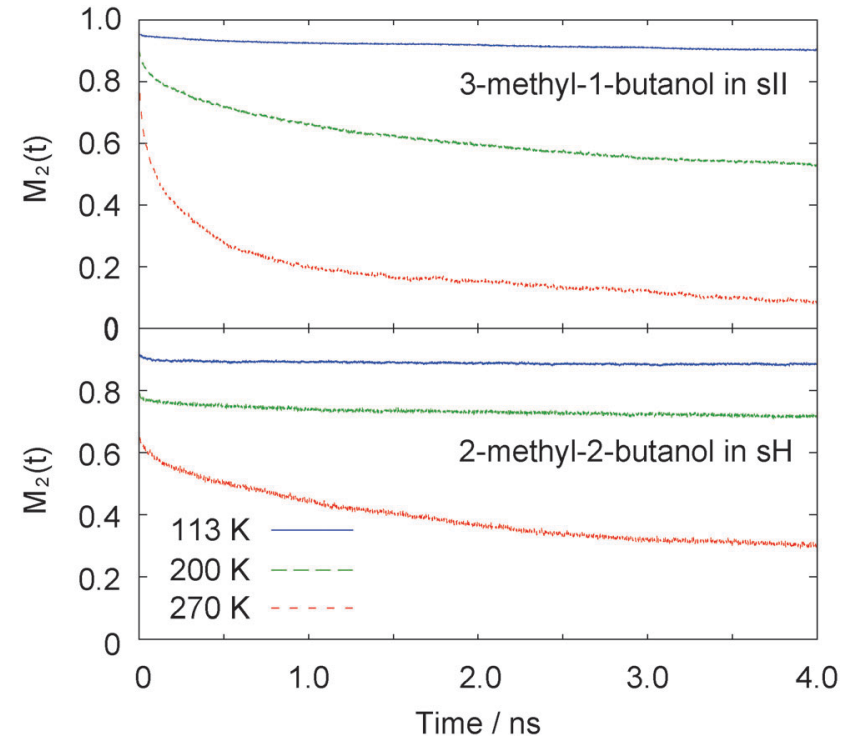

Fig. 6 Decay of the $M_{2}$ correlation functions with time for 3-methyl-1butanol in the sll (top) and 2-methyl-2-butanol in the sH hydrate (bottom). The relaxation time of $M_{2}$ in the sll hydrate is smaller than that in the $\mathrm{sH}$ hydrates at all temperatures. Decay of the $S_{\mathrm{HB}}$ with time for 3-methyl-1butanol in the sll (top) and 2-methyl-2-butanol in the sH hydrate (bottom).

fitting of exponential functions are shown in the ESI $\dagger$ ). To understand this trend, we calculate the continuous hydrogen bond time correlation function, $S_{\mathrm{HB}}(t),{ }^{63}$

$$
S_{\mathrm{HB}}(t)=\frac{\langle\mathrm{H}(0) \mathrm{H}(t)\rangle}{\left\langle\mathrm{H}(0)^{2}\right\rangle}
$$

where $\mathrm{H}(t)$ is the hydrogen bonding population variable defined such that $\mathrm{H}(t)=1$ if the guest-host hydrogen bond pair remains stable continuously from $t=0$ to time $t$ and it is zero otherwise (i.e., if the guest-host hydrogen bonding is broken or the pair is changed). The brackets represent ensemble averages over all guest molecules in the system. $S_{\mathrm{HB}}(t)$ describes the probability that guest-host hydrogen bond pair remains stable at all times up to $t$. Fig. 7 shows that the hydrogen bond correlation function, $S_{\mathrm{HB}}(t)$, of 3-methyl-1butanol in the sII $5^{12} 6^{4}$ cages decays faster than that of 2-methyl-2-butanol in the $\mathrm{sH} 5^{12} 6^{8}$ cages. Despite having greater hydrogen bonding probability, the guest-host hydrogen bond pairs for 3-methyl-1-butanol in the sII $5^{12} 6^{4}$ cages break and reform more frequently than those for the 2-methyl-2butanol in the $\mathrm{sH} 5^{12} 6^{8}$. This more frequent transition of the guest-host hydrogen bonding pair indicates the lower potential barrier of the transition and results in the faster decay of $M_{2}(t)$ for 3-methyl-1-butanol in the sII $5^{12} 6^{4}$ cages.

In the $10 \mathrm{~ns}$ of the classical MD simulation, we also observed guest-guest hydrogen bonds in the sII hydrate. Since all $5^{12} 6^{4}$ cages in sII hydrates share hexagonal faces with other $5^{12} 6^{4}$ cages, the rotating alcohol $\mathrm{O}-\mathrm{H}$ groups in the adjacent cages can be found close to each other. As shown in Table 1, about $4 \%$ of the alcohol molecules formed guest-guest hydrogen bonds through the hexagonal faces at $270 \mathrm{~K}$. The same geometric criteria of the $\mathrm{OH}_{\mathrm{g}} \cdots \mathrm{O}_{\mathrm{w}}$ distance and the $\mathrm{OH}_{\mathrm{g}} \cdots \mathrm{O}_{\mathrm{w}}$ angle as 


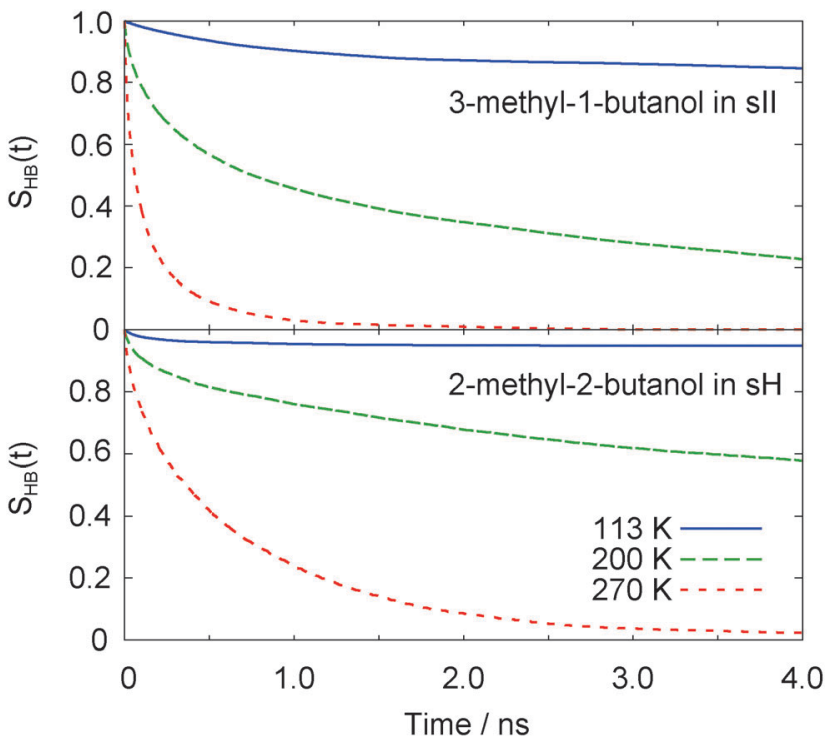

Fig. 7 Decay of the $S_{H B}$ with time for 3-methyl-1-butanol in the sll (top) and 2-methyl-2-butanol in the $\mathrm{sH}$ hydrate (bottom). The continuous time of the guest-host hydrogen bond pair of the sll 3-methyl-1-butanol hydrate is shorter than that of the $\mathrm{sH}$ 2-methyl-2-butanol hydrate.

used for guest-host hydrogen bonds were used to determine the presence of guest-guest hydrogen bonding. A snapshot of a guest-guest hydrogen bond in the cages is shown in Fig. 8. In previous studies, the guest-guest interactions have always been excluded when estimating the thermodynamic stability of the hydrate phase. However, as seen in this study, such guest-guest interactions can have important consequences in the hydrate stability.

We should mention that we used full occupancy of the small cages with methane molecules. Previous MD simulations reported that the relaxation time is affected by the occupancy of clathrate hydrates. ${ }^{59,64}$ More detailed analysis focusing on the occupancy and types of guest molecules should be performed in future considering varying degrees of occupancy of the small cages.

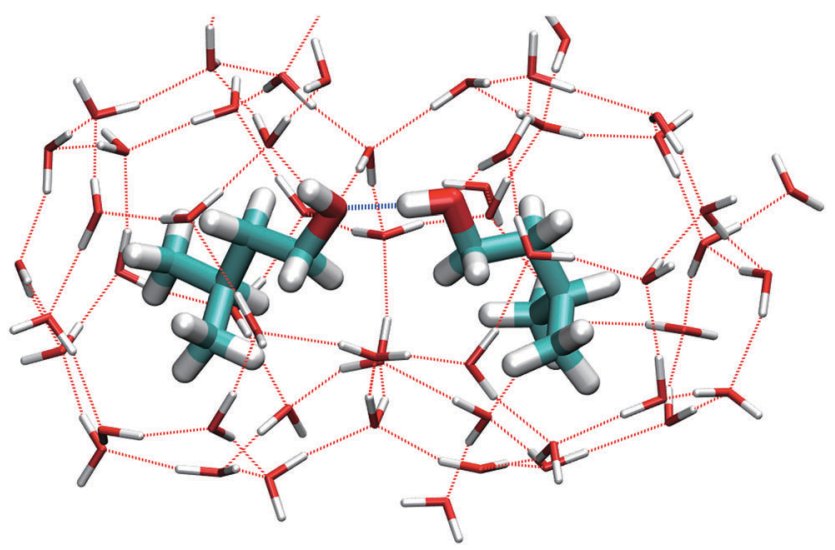

Fig. 8 Snapshot of the guest-guest hydrogen bond between 3-methyl1-butanol molecules in sll hydrate observed in the classical MD simulation at $270 \mathrm{~K}$. The guest-guest hydrogen bonding was occurred through a hexagonal face. The probability was about 0.04 .

\section{Conclusions}

$A b$ initio and classical molecular dynamics simulations were performed to study the effects of guest-host hydrogen bonding on the structure and stability of two amyl-alcohol clathrate hydrates. The simulations showed the formation of guest-host hydrogen bonds and large frequency shift of the O-H stretching vibration of the 3-methyl-1-butanol molecule in sII clathrate hydrate. The guest-host hydrogen bond was quite stable and the calculated $\mathrm{O}-\mathrm{H}$ vibrational frequency shift was consistent with previous experimental Raman spectra where the free $\mathrm{O}-\mathrm{H}$ stretching vibrational peak of amyl-alcohol in sII hydrate was not observed. ${ }^{16}$ The O-H group of 3-methyl-1-butanol molecules was incorporated into the water lattice of the $5^{12} 6^{4}$ cages. As a result, the large alcohol molecule can fit the relatively small $5^{12} 6^{4}$ cages. The gauche structure of 3-methyl-1-butanol molecules was observed in the sII $5^{12} 6^{4}$ cages and the effect of the conformation of the guest molecules on the cage stability was indicated. Guesthost hydrogen bonding was also observed in sH clathrate hydrate containing 2-methyl-2-butanol. Relatively smaller probability of the guest-host hydrogen bond and frequency shift of the $\mathrm{O}-\mathrm{H}$ stretching vibration than in the sII hydrate were observed in this case. About $15 \%$ of the hydrogen atoms in $\mathrm{O}-\mathrm{H}$ groups of the enclathrated 2-methyl-2-butanol molecules did not form hydrogen bonds at $113 \mathrm{~K}$ and this existence of a non-hydrogen bonded, free hydrogen atom of the $\mathrm{O}-\mathrm{H}$ group of alcohol molecules is consistent with Raman observations. ${ }^{16}$ The differences in the behavior of the guest-host hydrogen bonds were related to the location of the O-H group in the alcohol molecules. 3-Methyl-1butanol is primary alcohol and is easily incorporated into the hydrate cage. In contrast, 2-methyl-2-butanol is a tertiary alcohol with the $\mathrm{O}-\mathrm{H}$ group near the center of the molecule, making the formation of strong hydrogen bonds with the cage water molecules more difficult due to the steric effects from the adjacent methyl groups. Therefore, the 3-methyl-1-butanol molecules are enclosed in the $5^{12} 6^{4}$ in sII with methane while the 2-methyl-2butanol is observed in the larger $5^{12} 6^{8}$ cages in the sH hydrate. The position of the hydrophilic part of guest molecules plays an important role in determining the stability of the cages and the structure of the clathrate hydrate.

\section{Acknowledgements}

This work was supported by a Grant-in-Aid for JSPS Fellows (Grant No. 25-5399).

\section{References}

1 K. A. Kvenvolden, Proc. Natl. Acad. Sci. U. S. A., 1999, 96, 3420-3426.

2 F. Fleyfel and J. Devlin, J. Phys. Chem., 1991, 3811-3815.

3 W. L. Mao, H.-K. Mao, A. F. Goncharov, V. V. Struzhkin, Q. Guo, J. Hu, J. Shu, R. J. Hemley, M. Somayazulu and Y. Zhao, Science, 2002, 297, 2247-2249. 
4 L. J. Florusse, C. J. Peters, J. Schoonman, K. C. Hester, C. A. Koh, S. F. Dec, K. N. Marsh and E. D. Sloan, Science, 2004, 306, 469-471.

5 H. Lee, J.-W. Lee, D. Y. Kim, J. Park, Y.-T. Seo, H. Zeng, I. L. Moudrakovski, C. I. Ratcliffe and J. A. Ripmeester, Nature, 2005, 434, 743-746.

6 T. Nakajima, S. Akatsu, R. Ohmura, S. Takeya and Y. H. Mori, Angew. Chem., Int. Ed., 2011, 50, 10340-10343.

7 R. G. Grim, P. B. Kerkar, M. Shebowich, M. Arias, E. D. Sloan, C. A. Koh and A. K. Sum, J. Phys. Chem. C, 2012, 116, 18557-18563.

8 D.-Y. Koh, H. Kang, J. Jeon, Y.-H. Ahn, Y. Park, H. Kim and H. Lee, J. Phys. Chem. C, 2014, 118, 3324-3330.

9 J. H. van der Waals and J. C. Platteeuw, Adv. Chem. Phys., 1959, 2, 1-57.

10 E. D. Sloan, Nature, 2003, 426, 353-363.

11 D. Davidson, S. Gough, J. A. Ripmeester and H. Nakayama, Can. J. Chem., 1981, 59, 2587-2590.

12 B. Gbaruko, J. Igwe, P. Gbaruko and R. Nwokeoma, J. Pet. Sci. Eng., 2007, 56, 192-198.

13 K. Shin, R. Kumar, K. A. Udachin, S. Alavi and J. A. Ripmeester, Proc. Natl. Acad. Sci. U. S. A., 2012, 109, 14785-14790.

14 K. K. Stergaard, B. Tohidi, R. Anderson, A. C. Todd and A. Danesh, Ind. Eng. Chem. Res., 2002, 41, 2064-2068.

15 V. Buch, J. P. Devlin, I. A. Monreal, B. Jagoda-Cwiklik, N. Uras-Aytemiz and L. Cwiklik, Phys. Chem. Chem. Phys., 2009, 11, 10245-10265.

16 M. Cha, K. Shin and H. Lee, J. Phys. Chem. B, 2009, 113, 10562-10565.

17 M. Cha, K. Shin, M. Kwon, D.-Y. Koh, B. Sung and H. Lee, J. Am. Chem. Soc., 2010, 132, 3694-3696.

18 R. Ohmura, S. Takeya, T. Uchida, I. Y. Ikeda, T. Ebinuma and H. Narita, Fluid Phase Equilib., 2004, 221, 151-156.

19 K. Shin, K. A. Udachin, I. L. Moudrakovski, D. M. Leek, S. Alavi, C. I. Ratcliffe and J. A. Ripmeester, Proc. Natl. Acad. Sci. U. S. A., 2013, 110, 8437-8442.

20 J. P. Devlin, J. Chem. Phys., 2014, 140, 164505.

21 J. S. Loveday, R. J. Nelmes, M. Guthrie, S. A. Belmonte, D. R. Allan, D. D. Klug, J. S. Tse and Y. P. Handa, Nature, 2001, 410, 661-663.

22 S. Takeya, K. A. Udachin, I. L. Moudrakovski, R. Susilo and J. A. Ripmeester, J. Am. Chem. Soc., 2010, 132, 524-531.

23 T. Makiya, T. Murakami, S. Takeya, A. K. Sum, S. Alavi and R. Ohmura, Phys. Chem. Chem. Phys., 2010, 12, 9927-9932.

24 K. A. Udachin and J. A. Ripmeester, Nature, 1999, 397, 420-423.

25 K. Udachin, S. Alavi and J. A. Ripmeester, J. Chem. Phys., 2011, 134, 121104.

26 I. A. Monreal, L. Cwiklik, B. Jagoda-Cwiklik and J. P. Devlin, J. Phys. Chem. Lett., 2010, 1, 290-294.

27 R. Susilo, S. Alavi, S. Lang, J. A. Ripmeester and P. Englezos, J. Phys. Chem. C, 2008, 112, 9106-9113.

28 R. Susilo, S. Alavi, I. L. Moudrakovski, P. Englezos and J. A. Ripmeester, ChemPhysChem, 2009, 10, 824-829.

29 S. Alavi, S. Takeya, R. Ohmura, T. K. Woo and J. A. Ripmeester, J. Chem. Phys., 2010, 133, 074505.

30 S. Alavi and J. A. Ripmeester, J. Chem. Phys., 2012, 137, 54712.
31 T. M. Chang, R. J. Cooper and E. R. Williams, J. Am. Chem. Soc., 2013, 135, 14821-14830.

32 W. Lee, D. Lim and H. Lee, Electrochim. Acta, 2013, 109, 852-858.

33 Y. Jin, M. Kida and J. Nagao, J. Phys. Chem. C, 2013, 117, 23469-23475.

34 K. A. Udachin, S. Alavi and J. A. Ripmeester, J. Phys. Chem. C, 2013, 117, 14176-14182.

35 J. Zhu, S. Du, X. Yu, J. Zhang, H. Xu, S. C. Vogel, T. C. Germann, J. S. Francisco, F. Izumi, K. Momma, Y. Kawamura, C. Jin and Y. Zhao, Nat. Commun., 2014, 5, 4128.

36 J. A. Ripmeester and C. I. Ratcliffe, J. Phys. Chem., 1990, 94, 8773-8776.

37 S. Alavi, K. Udachin, C. I. Ratcliffe and J. A. Ripmeester, Supramol. Chem. From Mol. to Nanomater, 2012, pp. 1-16.

38 R. Car and M. Parrinello, Phys. Rev. Lett., 1985, 55, 2471-2474. 39 J. Hutter and M. Iannuzzi, Z. Kristallgr., 2005, 220, 549-551.

40 J. Perdew, K. Burke and M. Ernzerhof, Phys. Rev. Lett., 1996, 77, 3865-3868.

41 J. S. Tse, J. Supramol. Chem., 2002, 2, 429-433.

42 Q.-S. Du, P.-j. Liu, J. Deng and N. New, J. Chem. Theory Comput., 2007, 3, 1665-1672.

43 G. Román-Pérez, M. Moaied, J. Soler and F. Yndurain, Phys. Rev. Lett., 2010, 105, 8-11.

44 S. Grimme, J. Comput. Chem., 2006, 27, 1787-1799.

45 N. Troullier and J. L. Martins, Phys. Rev. B: Condens. Matter Mater. Phys., 1991, 43, 1993-2006.

46 L. Kleinman and D. M. Bylander, Phys. Rev. Lett., 1982, 48, 1425-1428.

47 S. W. Ong, E. S. Tok and H. C. Kang, Phys. Chem. Chem. Phys., 2010, 12, 14960-14966.

48 M. Hiratsuka, R. Ohmura, A. K. Sum and K. Yasuoka, J. Chem. Phys., 2012, 136, 44508.

49 M. Hiratsuka, R. Ohmura, A. K. Sum and K. Yasuoka, J. Chem. Phys., 2012, 137, 144306.

50 R. K. McMullan and G. A. Jeffrey, J. Chem. Phys., 1965, 42, 2725.

51 F. Takeuchi, M. Hiratsuka, R. Ohmura, S. Alavi, A. K. Sum and K. Yasuoka, J. Chem. Phys., 2013, 138, 124504.

52 D. Van Der Spoel, E. Lindahl, B. Hess, G. Groenhof, A. E. Mark and H. J. C. Berendsen, J. Comput. Chem., 2005, 26, 1701-1718.

53 B. Hess, C. Kutzner, D. van der Spoel and E. Lindahl, J. Chem. Theory Comput., 2008, 4, 435-447.

54 J. L. F. Abascal, E. Sanz, R. García Fernández, C. Vega and R. Garcá Fernández, J. Chem. Phys., 2005, 122, 234511.

55 M. M. Conde and C. Vega, J. Chem. Phys., 2010, 133, 064507. 56 W. D. Cornell, P. Cieplak, C. I. Bayly, I. R. Gould, K. M. Merz, D. M. Ferguson, D. C. Spellmeyer, T. Fox, J. W. Caldwell and P. A. Kollman, J. Am. Chem. Soc., 1995, 117, 5179-5197.

57 M. J. Frisch, G. W. Trucks, H. B. Schlegel, G. E. Scuseria, M. A. Robb, J. R. Cheeseman, J. A. Montgomery, Jr., T. Vreven, K. N. Kudin, J. C. Burant, J. M. Millam, S. S. Iyengar, J. Tomasi, V. Barone, B. Mennucci, M. Cossi, G. Scalmani, N. Rega, G. A. Petersson, H. Nakatsuji, M. Hada, M. Ehara, K. Toyota, R. Fukuda, J. Hasegawa, M. Ishida, T. Nakajima, Y. Honda, O. Kitao, H. Nakai, M. Klene, X. Li, J. E. Knox, H. P. Hratchian, J. B. Cross, V. Bakken, C. Adamo, J. Jaramillo, R. Gomperts, R. E. Stratmann, O. Yazyev, A. J. Austin, 
R. Cammi, C. Pomelli, J. W. Ochterski, P. Y. Ayala, K. Morokuma, G. A. Voth, P. Salvador, J. J. Dannenberg, V. G. Zakrzewski, S. Dapprich, A. D. Daniels, M. C. Strain, O. Farkas, D. K. Malick, A. D. Rabuck, K. Raghavachari, J. B. Foresman, J. V. Ortiz, Q. Cui, A. G. Baboul, S. Clifford, J. Cioslowski, B. B. Stefanov, G. Liu, A. Liashenko, P. Piskorz, I. Komaromi, R. L. Martin, D. J. Fox, T. Keith, M. A. AlLaham, C. Y. Peng, A. Nanayakkara, M. Challacombe, P. M. W. Gill, B. Johnson, W. Chen, M. W. Wong, C. Gonzalez and J. A. Pople, Gaussian 03, RevisionC.02, Gaussian, Inc., Wallingford, CT, 2004.

58 S. Alavi, R. Ohmura and J. A. Ripmeester, J. Chem. Phys., 2011, 134, 054702.
59 S. Alavi, K. Udachin and J. A. Ripmeester, Chem. - Eur. J., 2010, 16, 1017-1025.

60 T. J. Frankcombe and G.-J. Kroes, Phys. Chem. Chem. Phys., 2011, 13, 13410-13420.

61 N. Bloembergen, E. Purcell and R. Pound, Phys. Rev., 1948, 73, 679-712.

62 H. Mohammadi-Manesh, S. Alavi, T. K. Woo and B. Najafi, Phys. Chem. Chem. Phys., 2011, 13, 2367-2377.

63 J. R. Choudhuri, V. K. Yadav, A. Karmakar, B. S. Mallik and A. Chandra, Pure Appl. Chem., 2013, 85, 27-40.

64 A. T. Trueba, M. C. Kroon, C. J. Peters, I. L. Moudrakovski, C. I. Ratcliffe, S. Alavi and J. A. Ripmeester, J. Chem. Phys., 2014, 140, 214703. 